\title{
Simulation and Experimental Investigation of Automotive Disc Brakes for 150CC Pulsar Bike
}

\author{
Prashant C. Jadhav* and Sandip. H. Deshmukh \\ Mechanical Engineering, Savitribai Phule Pune University, Pune, India \\ Accepted 15 June 2016, Available online 20 June 2016, Special Issue-5 (June 2016)
}

\begin{abstract}
Various parameters affect performance of disc, out of those three main parameters are pressure applied on pad, coefficient of friction at pad-disc interface and temperature generated due to friction at interface. It was decided to check these parameter effect by simulating them using Explicit Dynamics code LS-Dyna. Simulation is effective in a way as the cycle time for final product design and its validation can be reduced significantly. Also it aids to reduced prototyping cost. Simulation and Experimentation was carried out to monitor the effect of these parameters for structural and thermal analysis of disc brakes. The selected disc brake under study is of Pulsar 150 Bike. The Simulation and Analytical outcomes of the project are presented in the paper along with experimental validation planned in course.
\end{abstract}

Keywords: Thermal Analysis, Structural Analysis, Disc brake.

\section{Introduction}

Nowadays bikes and cars uses a combination of disc brakes and drum brakes. For car, normally disc brakes are on the front two wheels and drum brakes on the rear wheels and in case of bikes disc brake on front and drum brake on rear. However, the performance of braking system depends on the design and selection of material. Three functions that must be complied by the brakes for all the time are:

1) The braking system should reduce the speed of a vehicle in a controlled fashion and when appropriate brings the vehicle to stop.

2) The braking must allow the vehicle to maintain a constant speed when travelling downhill.

3) The braking system should hold the vehicle stationary when on the flat or on a gradient.

Study of moment of inertia is done to simulate the realistic brake process instead of theoretically predefines the train deceleration rate, nonlinear deceleration rate and thermo-mechanical behavior has been revealed (Jiguang Chen, 2014). A coupled numerical-experimental approach was studied to determine the critical thermo mechanical loadings associated with braking induced metallurgical phase transformations (M. Collignon et al, 2013). The results of the braking numerical simulations on a pegs-wing

*Corresponding author: Prashant C. Jadhav ventilated disk brake rotor, obtained by performing a fading braking procedure and the results are compared with the experimental ones obtained in the same brake, mounted on a work bench, in the same fading braking procedure (Pier Francesco Gotowicki et al, 2005). The generated methodology adopted for determining the thermal and structural boundary conditions which is determined experimentally and validated by FEA for a two wheeler drum brake. (O.P.Singh et al, 2010). The frictional heat generated during braking application can cause numerous negative effects on the brake assembly such as brake fade, premature wear, thermal cracks and Disc Thickness Variation (DTV). In the past, surface roughness and wear at the pad interface have rarely been considered in studies of thermal analysis of a disc brake assembly using finite element method. The ventilated pad-disc brake assembly is built by a 3D model with a thermo-mechanical coupling boundary condition and multi-body model technique.

The numerical simulation for the coupled transient thermal field and stress field is carried out by sequentially thermal-structural coupled method based on ANSYS to evaluate the stress fields and of deformations (D. Murali Mohan Rao et al, 2013). Temperature fields and structural fields of the solid disc brake during short and emergency braking with four different materials was Investigated. The distribution of the temperature depends on the various factors such as friction, surface roughness and speed. The effect of the angular velocity and the contact 
pressure induces the temperature rise of disc brake. The finite element simulation for two-dimensional model was preferred due to the heat flux ratio constantly distributed in circumferential direction. The values of temperature, friction contact power, nodal displacement and deformation for different pressure conditions were evaluated using analysis software with four materials namely cast iron, cast steel, aluminum and carbon fiber reinforced plastic. Presently the Disc brakes are made up of cast iron and cast steel. With the value at the hand best suitable material for the brake drum with higher life span can be determined (Daniel Das.A et al, 2013). A new method to evaluate thermal fatigue by a simulating high-speed braking test using an actual disc brake rotor was studied. Thermal fatigue strength is confirmed to be improved with increasing graphite number in the microstructure. It is also confirmed that the graphite number increases in proportion to the amount of nickel added, and that the inoculation of cerium, a rare earth element, produces an effect similar to that of adding nickel. Based on this approach, a new, low cost material for disc brake rotors for heavy- and medium-duty trucks is developed using both nickel and cerium(Junichiro Yamabe et al, 2003). Hence it was decided to carry out analysis to study the effect of pad pressure, coefficient of friction at pad-disc interface and temperature effect due to friction on disc performance.

\section{Scope}

1) Thermal and Structural Analysis of Disc Brake Rotor.

2) Mathematical model generation for Brake Calculation.

3) Comparison of Mathematical and Simulation model.

\section{Modeling and Analysis}

It is not easy to exactly model the brake disc, in which still researches are going on to find out brake behavior during braking applications. In modeling we always ignore the things that are of less importance and have little impact on the analysis. The assumptions which are made are given below:

1) Rotor Disc material is assumed as homogeneous and isotropic.

2) The system is assumed as axis-symmetric.

3) Inertia and body force effects are bare minimum during the analysis.

4) The disc is assumed to be stress free before the application of brake.

5) The analysis does not determine the life of the disc brake.

6) Only ambient air-cooling is taken into account and no forced Convection is taken.

7) The kinetic energy of the vehicle is lost through the brake discs i.e. no heat loss between the tyre and the road surface and deceleration is uniform.

8) The disc brake model used is of solid type and not ventilated one.

9) The thermal conductivity and specific heat of the material used for the analysis is uniform throughout and does not change with temperature.

10) No disc wear is considered in analysis.

\subsection{CAD Details}

The CAD model is created using PRO-E 4.0. The dimensions are measured from the existing disc brake of Bajaj pulsar 150. Pro/ENGINEER Wildfire is a standard 3D product design software, featuring industry-leading productivity tools that promote best practices in design while ensuring compliance with industry standards.

The Disc Brake details are as follows

$\begin{array}{ll}\text { Material } & : \text { Stainless Steel } \\ \text { Outer Diameter } & : 240 \mathrm{~mm} \\ \text { Inner Diameter } & : 110 \mathrm{~mm} \\ \text { Thickness } & : 5 \mathrm{~mm} \\ \text { Mean Contact Radius } & : 107.5 \mathrm{~mm} \\ \text { Hole Diameter } & : 7 \mathrm{~mm}\end{array}$

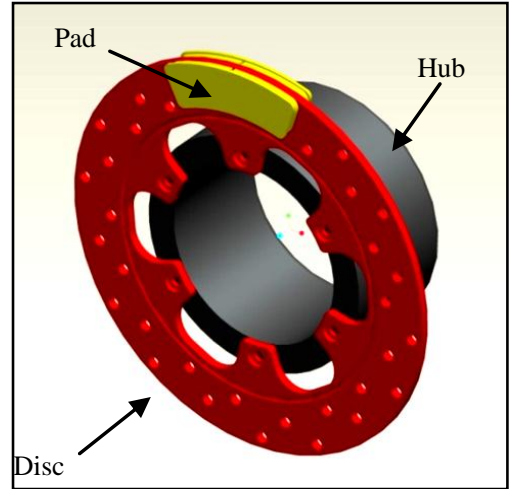

Fig.1 Disc Brake Assembly

\subsection{Meshing Details}

Mesh generation was done using Hypermesh 11.0. The element types chosen are Hexahedron (brick) element and Tetrahedron element.

Table 1 Mesh Details

\begin{tabular}{|c|c|c|}
\hline & No. of Elements & No. of Nodes \\
\hline Meshed model & 18134 & 15702 \\
\hline
\end{tabular}

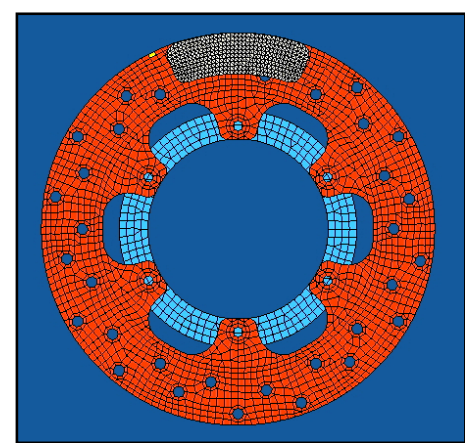

Fig.2 Meshed model of Assembly 


\subsection{Material Properties}

The material properties considered for pad and disc are:

Table 2 Disc Pad Material properties

\begin{tabular}{|c|c|}
\hline Density & $2500 \mathrm{Kg} / \mathrm{m}^{3}$ \\
\hline Thermal conductivity & $12 \mathrm{~W} / \mathrm{m} . \mathrm{K}$ \\
\hline Specific Heat Capacity & $900 \mathrm{~J} / \mathrm{Kg} . \mathrm{K}$ \\
\hline Poisson's ratio & 0.3 \\
\hline
\end{tabular}

Table 3 Disc Material properties

\begin{tabular}{|c|c|}
\hline Property & Material 410 \\
\hline Density & $7750 \mathrm{Kg} / \mathrm{m}^{3}$ \\
\hline Thermal conductivity & $43 \mathrm{~W} / \mathrm{m} . \mathrm{K}$ \\
\hline Specific Heat Capacity & $460 \mathrm{~J} / \mathrm{Kg} \cdot \mathrm{K}$ \\
\hline Coefficient of Thermal & \\
Expansion & \\
20 to $200^{\circ} \mathrm{C}$ & $10.5 \times 10-6 /{ }^{\circ} \mathrm{C}$ \\
200 to $600^{\circ} \mathrm{C}$ & $11.6 \times 10-6 /{ }^{\circ} \mathrm{C}$ \\
\hline Modulus of Elasticity & $200 \mathrm{GPa}$ \\
\hline Poisson's ratio & 0.3 \\
\hline Shear Modulus & $80 \mathrm{GPa}$ \\
\hline Tensile Strength Ultimate & $450 \mathrm{MPa}$ \\
\hline Tensile Strength Yield & $415 \mathrm{MPa}$ \\
\hline
\end{tabular}

\subsection{Evaluation of Input parameters}

For evaluation of Input parameters following empirical relationships were used. As per Bureau of Indian standards (IS-13453.1994) the purpose of the Brake Inertia Dynamometer is to conduct various tests for performance and durability for the Two Wheeler Disc Brake System like:

Type D1 Test (Performance Test)

Type D2 Test (Fade Test)

Type D3 Test (Wet / Dry Test)

Type D2 Test:

Requirement of Brake Dynamometer the brake dynamometer shall be capable of adjustment to the inertia settings specified below, the inertia setting for the front and rear wheel shall be obtained by the formula

where,

$$
\mathrm{I}=\frac{\mathrm{WR}^{2}}{\mathrm{~g}} \times \frac{1}{\mathrm{~N}}
$$

I = Inertia in kg.m.s ${ }^{2}$

$\mathrm{W}=$ the mass of vehicle to be simulated in $\mathrm{kg}=205 \mathrm{Kg}$

$\mathrm{R}=$ static load radius of tyre of the wheel where the brake

under test is normally fitted in $\mathrm{m}=0.3 \mathrm{~m}$

$\mathrm{g}=$ acceleration due to gravity $=9.81 \mathrm{~m} / \mathrm{s} 2$

$\mathrm{N}$ = number of brakes normally fitted on the wheel $=1$

$I=\frac{205 \times 0.3^{2}}{9.81} \times \frac{1}{1}$
$\mathrm{I}=1.88 \mathrm{Kg} \cdot \mathrm{m} \cdot \mathrm{s}^{2}$

$\mathrm{I}=18.44 \mathrm{Kg} \cdot \mathrm{m}^{2}$

To simulate the inertia of flywheel the hub density $(\rho)$ was calculated to be $8821.8 \mathrm{E}+03 \mathrm{Kg} / \mathrm{m}^{3}$

The units considered are as per the formulation and Simulation software requirement. The simulation parameters were -

Table 4 Simulation Parameters

\begin{tabular}{|c|c|c|c|}
\hline Description & Notation & Value & Unit \\
\hline Flywheel Inertia & $\mathrm{I}$ & 18.44 & $\mathrm{Kg} . \mathrm{m}^{2}$ \\
\hline $\begin{array}{c}\text { Pressure in fluid } \\
\text { system }\end{array}$ & $\mathrm{P}$ & 35 & $\mathrm{Bar}$ \\
\hline $\begin{array}{c}\text { Coefficient of } \\
\text { Friction }\end{array}$ & $\mathrm{R}$ & 0.35 & - \\
\hline Radius of wheel & $\mathrm{R}$ & 0.3 & $\mathrm{~m}$ \\
\hline $\begin{array}{c}\text { Initial velocity of } \\
\text { vehicle }\end{array}$ & $\mathrm{V}$ & 60 & $\mathrm{Kmph}$ \\
\hline Angular velocity & $\alpha$ & 55.5 & $\mathrm{rad} / \mathrm{s}$ \\
\hline $\begin{array}{c}\text { Pressure Applied } \\
\text { on pad }\end{array}$ & $\mathrm{P}_{\mathrm{pad}}$ & 2 & $\mathrm{MPa}$ \\
\hline $\begin{array}{c}\text { Assigned Hub } \\
\text { density }\end{array}$ & $\rho$ & $\begin{array}{c}8821.8 \mathrm{E}+0 \\
3\end{array}$ & $\mathrm{Kg} / \mathrm{m}^{3}$ \\
\hline
\end{tabular}

\section{Calculation}

For Velocity of vehicle $=60 \mathrm{kmph}=16.67 \mathrm{~m} / \mathrm{s}$

Considering deceleration of, $\mathrm{g}=4.6 \mathrm{~m} / \mathrm{s}^{2}$

Mass $=205 \mathrm{Kg}$

1) Time for stopping vehicle

$t=\frac{v}{g}=\frac{16.67}{4.6}=3.62 \mathrm{sec}$

2) Work done against friction or the heat available for dissipation can be calculated by

$$
\begin{aligned}
\mathrm{E}_{\text {diss }} & =0.5 \times \mathrm{I} \times \omega^{2} \\
& =0.5 \times 18.44 \times 55.5^{2} \\
& =28.4 \mathrm{KJ}
\end{aligned}
$$

3) The area of rubbing surface can be calculated as

$$
\begin{aligned}
A & =\pi\left(R_{2}^{2}-R_{1}^{2}\right) \\
& =\pi\left(118.5^{2}-93.5^{2}\right) \\
& =16650.44 \mathrm{~mm}^{2} \\
& =0.01665 \mathrm{~m}^{2}
\end{aligned}
$$

The rubbing surface is on both sides of brake disc, so

$$
\begin{aligned}
A= & 2 \times 0.01665 \\
& =0.03333 \mathrm{~m}^{2}
\end{aligned}
$$

4) Heat Flux $\left(Q_{f}\right)$

$Q_{f}=\frac{E_{\text {diss }}}{t} \times \frac{1}{A}$

$Q_{f}=\frac{28400}{3.62} \times \frac{1}{0.0333}$ 


$$
=235.594 \mathrm{KW} / \mathrm{m}^{2}
$$

5) Single stop temperature rise can be calculated as

$T s=\left(\frac{0.527 \times Q_{f} \times \sqrt{t}}{\sqrt{\left(\rho \times C_{p} \times k\right)}}\right)$

$T s=\left(\frac{0.527 \times 235594 \times \sqrt{3.62}}{\sqrt{(7750 \times 460 \times 43)}}\right)$

$T s=19.08^{\circ} \mathrm{K}$

Final temperature can be calculated as

$$
\begin{aligned}
& T=T_{s}+T_{a m b} \\
& T=19.08+300=319.08^{\circ} \mathrm{K}=46.08^{\circ} \mathrm{C}
\end{aligned}
$$

6) Compressive stress developed in surface due to sudden temperature increase

$$
\begin{aligned}
\sigma & =\frac{E}{1-v} \times \alpha \times \Delta T \\
\sigma & =\frac{200000}{1-0.3} \times 10.5 \times 10^{\wedge}-6 \times 19.08 \\
\sigma & =57.24 \mathrm{MPa}
\end{aligned}
$$

\section{Results and Discussion}

A node was selected at mean radius of contact zone of bake pad with disc (Fig.4) and temperature contour plot was generated (Fig.5)

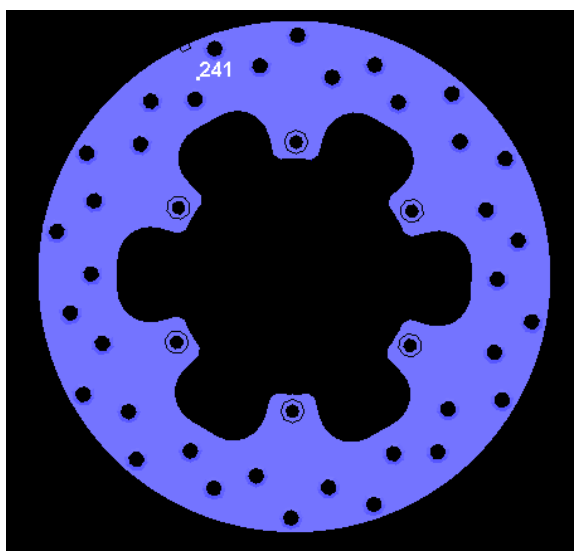

Fig.4 Node location on disc

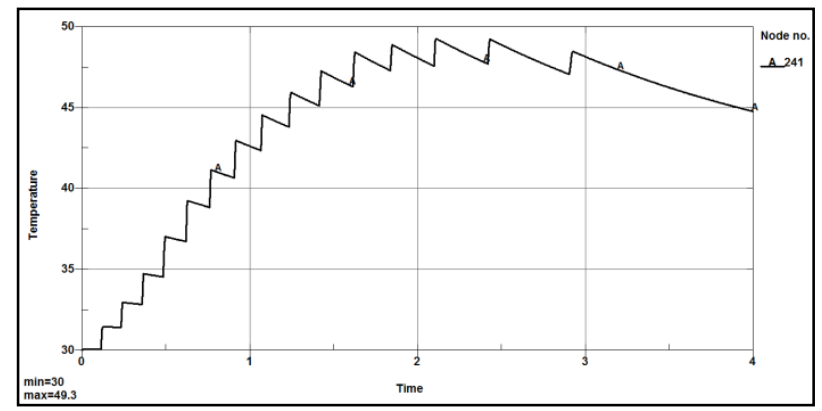

Fig.5 Nodal Temperature Plot
The temperature curves have a saw like shape which starts from the mutual rotational motion of the disc with respect to fixed pad. The presented temperature plot is obtained for certain fixed spot on the circumference of the disc, therefore periods of heating and cooling can be distinguished. The increase of temperature is because of accumulation of the frictional heat. On the other hand when the pad is out of considered spot on the rubbing path the cooling condition is established and the temperature decreases.

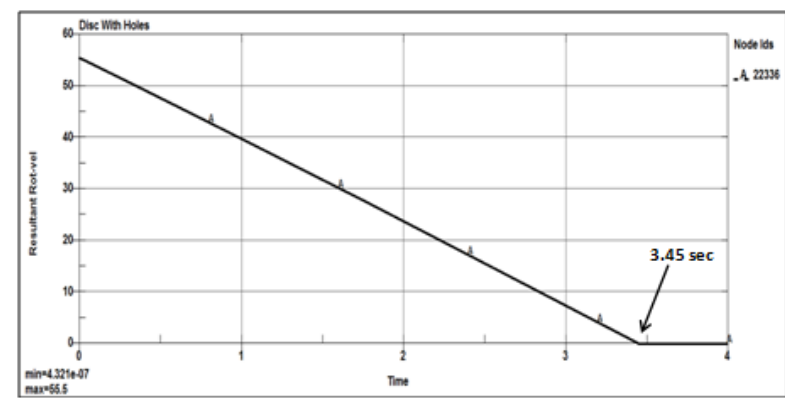

Fig.6 Resultant Rotational velocity of Disc

It can be observed that with application of constant pressure of $2 \mathrm{MPa}$ on brake pad the resultant rotational velocity reduces gradually from $55.5 \mathrm{rad} / \mathrm{s}$ to $0 \mathrm{rad} / \mathrm{s}$ and stopping time observed is $3.45 \mathrm{sec}$ (Fig.6).

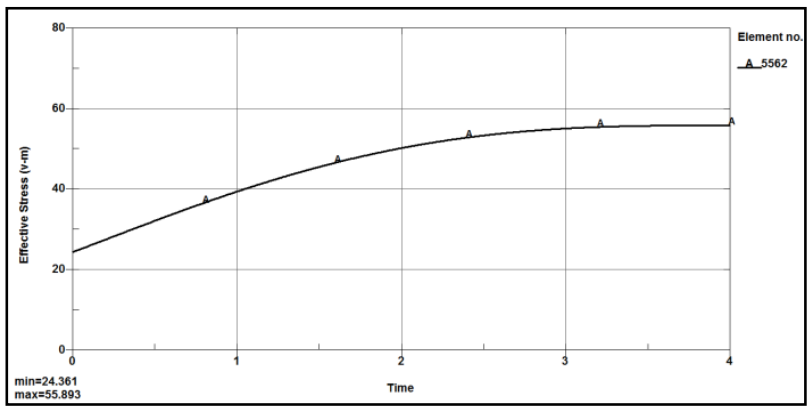

Fig.7 Stress developed due to temperature rise

The stress developed due to sudden temperature increase was measured at max. temperature location across the contact area and the stress value observed was 55.9 MPa (Fig.7).

Table 5 Analysis Result

\begin{tabular}{|c|c|c|c|}
\hline & Analysis Data & Calculation & Unit \\
\hline Avg. Stopping Time & 3.45 & 3.62 & $\mathrm{Sec}$ \\
\hline Avg. Deceleration & 4.83 & 4.6 & $\mathrm{~m} / \mathrm{s}^{2}$ \\
\hline $\begin{array}{c}\text { Max. Temperature } \\
\text { Rise }\end{array}$ & 49.3 & 46.08 & ${ }^{\circ} \mathrm{C}$ \\
\hline Stress & 55.89 & 57.24 & $\mathrm{MPa}$ \\
\hline
\end{tabular}




\section{Further work}

Testing of disc brake will be performed to study the parameters like stopping time, deceleration, max. temperature at pad-disc interface and these parameters will be compared with analysis results so that analysis methodology can be validated.

\section{Conclusion}

Disc brake model Analysis was performed using LSDyna solver code for stopping time of disc, deceleration, Max. Temperature at contact surface and stress due to sudden temperature rise. The temperature obtained at contact is computed by the specified angular velocity, pressure applied on brake pad and coefficient of friction at contact interface. The study was performed by considering mentioned loads and boundary conditions and by changing these parameters we can further study its effect on disc brake.

\section{References}

Jiguang Chen, Fei Gao (2014), Thermo-Mechanical Simulation of Brake Disc Frictional Character by Moment of Inertia, Research Journal of Applied Science Engineering and Technology 7(2), 227-232.

M. Collignon , L.Cristol, P.Dufrenoy, Y.Desplanques, D.Balloy (2013), "Failure of truck brake discs: A coupled numericalexperimental approach to identifying critical thermomechanical loadings, Tribology International 59, $114-120$.
Pier Francesco Gotowicki, Vinzenco Nigrelli, Gabriele Virzi Mariotti, Dr. Cedomir Duboka (2005), Numerical And Experimental Analysis Of A Pegs-Wing Ventilated Disk Brake Rotor, With Pads And Cylinders, 10th EAEC European Automotive Congress.

Adam Adamowicz, Piotr Grzes (2011), Analyzed disc brake temperature distribution during single braking under nonaxissymmetric load, Applied Thermal Engineering 31, 1003-1012.

O.P. Singh et al (2010), Thermal seizures in automotive drum brakes, Engineering Failure Analysis 17, 1155-1172.

S Naveen Kumar et al (2012) Redesign of Disc Brake Assembly with Lighter Material, International Journal of Engineering Research \& Technology (IJERT), ISSN: 22780181, Vol. 1 Issue 7.

Daniel Das.A et al (2013), Structural and Thermal Analysis of Disc Brake in Automobiles, International Journal of Latest Trends in Engineering and Technology (IJLTET), ISSN: 2278-621X, Vol. 2 Issue 3.

D. Murali Mohan Rao, Dr. C. Prasad, T. Ramakrishna (2013), Experimental and Simulated Studies on Temperature Distribution for Various Disc Brakes, International Journal of Research in Mechanical Engineering \& Technology (IJRMET) Vol. 3, Issue 1, ISSN : 2249-5762 (Online) | ISSN : 2249-5770 (Print), Nov - April.

Junichiro Yamabe, Masami Takagi, Toshiharu Matsui (2003), Development of Disc Brake Rotors for Heavy- and MediumDuty Trucks with High Thermal Fatigue Strength, Material Engineering Dept., Research \& Dev. Office, MFTBC, 2003.

Rudolf Limpert, Brake Design and Safety, Second Edition, SAE International. 